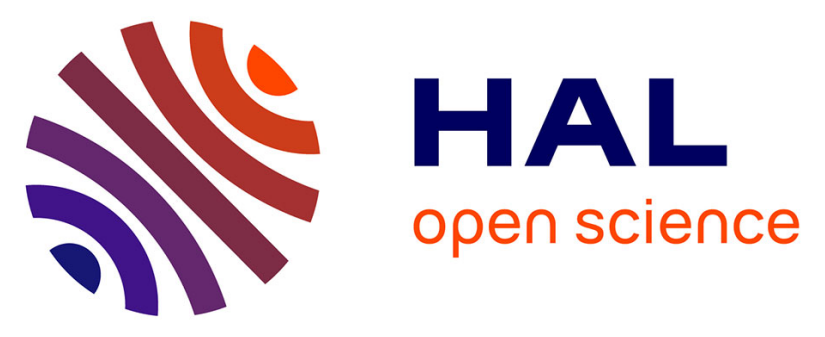

\title{
Ultrafast Ultrasound Plane Wave Imaging As a Novel non-Invasive Technique to Assess Diaphragm Contractility in Response to Phrenic Nerve Magnetic Stimulation
}

Thomas Poulard, Martin Dres, Marie-Cecile Nierat, Jean-Yves Hogrel, Thomas Similowski, Damien Bachasson, Jean-Luc Gennisson

\section{To cite this version:}

Thomas Poulard, Martin Dres, Marie-Cecile Nierat, Jean-Yves Hogrel, Thomas Similowski, et al.. Ultrafast Ultrasound Plane Wave Imaging As a Novel non-Invasive Technique to Assess Diaphragm Contractility in Response to Phrenic Nerve Magnetic Stimulation. 2020 IEEE International Ultrasonics Symposium (IUS), Sep 2020, Las Vegas, United States. pp.1-4, 10.1109/IUS46767.2020.9251595 . hal-03096304

\section{HAL Id: hal-03096304 https://hal.science/hal-03096304}

Submitted on 4 Jan 2021

HAL is a multi-disciplinary open access archive for the deposit and dissemination of scientific research documents, whether they are published or not. The documents may come from teaching and research institutions in France or abroad, or from public or private research centers.
L'archive ouverte pluridisciplinaire HAL, est destinée au dépôt et à la diffusion de documents scientifiques de niveau recherche, publiés ou non, émanant des établissements d'enseignement et de recherche français ou étrangers, des laboratoires publics ou privés. 


\title{
Ultrafast Ultrasound Plane Wave Imaging As a Novel non-Invasive Technique to Assess Diaphragm Contractility in Response to Phrenic Nerve Magnetic Stimulation
}

\author{
Thomas Poulard ${ }^{1,2}$, Martin Dres ${ }^{3,4}$, Marie-Cécile Niérat ${ }^{3}$, Jean-Yves Hogrel ${ }^{2}$, Thomas Similowski ${ }^{3,4}$, Damien \\ Bachasson $^{2 \#}$, Jean-Luc Gennisson ${ }^{1 \#}$ \\ \# equally contributing authors \\ ${ }^{1}$ Laboratoire d'Imagerie Biomédicale Multimodale, BioMaps, Université Paris-Saclay, CEA, CNRS UMR 9011, Inserm \\ UMR1281, SHFJ, 4 place du général Leclerc, 91401, Orsay, France \\ ${ }^{2}$ Institute of Myology, Neuromuscular Investigation Center, Neuromuscular Physiology Laboratory, Paris, France \\ ${ }^{3}$ Sorbonne Université, INSERM, UMRS1158 Neurophysiologie respiratoire expérimentale et clinique, Paris, France \\ 4 AP-HP. Sorbonne Université, Hôpital Pitié-Salpêtrière, Service de Pneumologie, Médecine intensive - Réanimation \\ (Département "R3S"), F-75013, Paris, France
}

\begin{abstract}
Diaphragm monitoring relies on the measurement of transdiaphragmatic pressure (Pdi) during cervical magnetic stimulation (CMS). Specifically, twitch Pdi $\left(\right.$ Pdi $\left._{\text {tw }}\right)$ is measured using esophageal and gastric probes. Such probes are invasive and require a high level of expertise, and non-invasive methods to assess diaphragm function are necessary. Here we test how maximal diaphragm tissue velocity $\left(\mathrm{Vdi}_{\max }\right)$ is related to stimulation intensity and to Pdi $_{t w}$ during CMS. Ultrafast ultrasound of the diaphragm was performed in 13 healthy subjects at various intensity levels. We show that $\mathrm{Vdi}_{\max }$ is strongly related to stimulation intensity and to $\mathbf{P d i}_{t w}$ in all participants. Within-day reliability for $\mathrm{Vdi}_{\max }$ was strong and similar to that of $\mathbf{P d i}_{t_{w}}$. Our findings opens prospect for the noninvasive monitoring of $\mathbf{P d i}_{t w}$ in clinical populations. Indeed, the relative changes in $\mathrm{Pdi}_{\mathrm{tw}}$ over time may be monitored by assessing the relative changes in $\mathrm{Vdi}_{\max }$.
\end{abstract}

Keywords-ultrafast ultrasound imaging, diaphragm, speckle tracking, cervical magnetic stimulation, contractility, skeletal muscle

\section{INTRODUCTION}

The diaphragm is the main respiratory muscle. Diaphragm pressure generation, namely the transdiaphragmatic pressure (Pdi), is defined as the difference between gastric (Pga) and esophageal (Pes) pressures. Specifically, twitch Pdi $\left(\mathrm{Pdi}_{\mathrm{tw}}\right)$ elicited by cervical magnetic stimulation (CMS) is the reference method to assess diaphragm contractility. However, measuring Pes and Pga, and therefore Pdi, relies on the use of invasive probes and requires a high level of expertise [1]. In this context, ultrasound (US) imaging of the diaphragm emerged as a relevant tool to assess diaphragm contractility.

To this end, various imaging technique have been used to evaluate diaphragm function. Diaphragm thickening fraction

Supported by the "Association Française contre les Myopathies" (AFM) and the "Fondation EDF"
[2] and strain [3] have been shown to be related to the changes in Pdi during breathing. Recently, our team showed that changed in diaphragm stiffness, measured using US shear wave elastography (SWE), were related to the changes in Pdi, both in healthy subjects and mechanically ventilated patients $[4,5]$. Nonetheless, these imaging methods provide a relatively low frame rate (from $2 \mathrm{~Hz}$ for SWE to $\sim 60 \mathrm{~Hz}$ for conventional US imaging). In turn, they cannot be used to image transient phenomena such as diaphragm behavior following CMS $(\sim 300 \mathrm{~ms})$. Ultrafast US allows very high frame rate (up to 20000 frames. $\mathrm{sec}^{-1}$ ) and may be used to image rapid contractions [6]. Ultrafast US enables the quantification of transient velocities of mechanical waves [7]. Previous work has reported that maximal tissue velocity within the biceps brachii increased linearly with stimulation intensity [8], but the potential link between tissue velocity and muscle force has not been reported.

The aims of this study were twofold: i) image the diaphragm during CMS at different intensity levels using ultrafast US and ii) investigated the relationship between indices derived from ultrafast US and $\mathrm{Pdi}_{\mathrm{tw}}$.

\section{MATERIALS \& METHODS}

\section{A. Subjects}

Thirteen healthy participants (5 males and 8 females, median $(\mathrm{Q} 1-\mathrm{Q} 3)-$ age $=24(22-27)$ years, height $=171(167-$ $183) \mathrm{cm}, \mathrm{BMI}=20.6(19.7-22.6) \mathrm{kg} \cdot \mathrm{m}-2)$ took part in this study. They were informed about the nature, aims and risks associated with the experiment before giving their written informed consent to participate. All participants were 18 and over with no history of neuromuscular and/or respiratory disorders, and no contraindications to CMS [9]. 


\section{B. Ultrafast Ultrasound Imaging}

A linear transducer (2-10 MHz, SL10-2) driven by an ultrafast ultrasound device (Aixplorer V12, Supersonic Imagine, Aix-en-Provence, France) was used to image the zone of apposition of the right hemidiaphragm. The probe was place at the $8^{\text {th }}-10^{\text {th }}$ intercostal space and the site of the probe was skin-marked to ensure that the same region was imaged across all recordings. Since diaphragm response following CMS is very brief $(\sim 300 \mathrm{~ms})$, a custom sequence was developed. A 9 angles $\left(-7^{\circ}\right.$ to $7^{\circ}$ with a $2^{\circ}$ incremental steps) plane-wave sequence at $9 \mathrm{kHz}$ was used, yielding a compounded frame rate of $1 \mathrm{kHz}$ a $500 \mathrm{~ms}$ acquisition [10]. The diaphragm could be

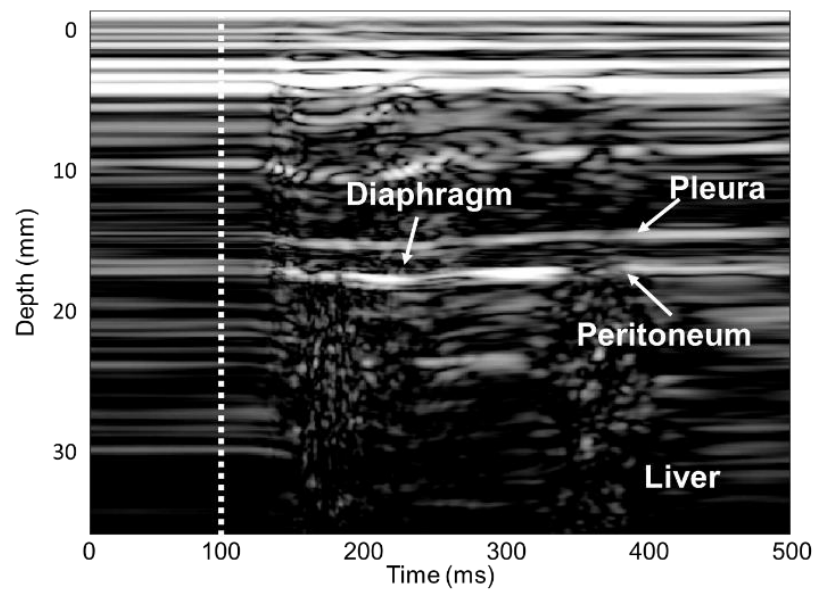

Fig. 1: Motion Mode (M-Mode) imaging of the diaphragm during cervical magnetic stimulation (CMS). CMS occurs at the $100 \mathrm{~ms}$ mark. The diaphragm is identified as a muscular layer surrounded by the pleura and peritoneum layers.

identified as a three layers structures superficial to the liver (Fig. 1).

\section{Pressure Measurements}

Pes and Pga were measured using $8 \mathrm{~cm}$ balloon catheters (Marquat Genie Biomedical, Boissy-Saint-Léger Cedex, France). Pdi was computed as the difference between Pga and Pes. Balloons were connected to differential pressures transducers (MLT0380/D, ADInstruments, Bella Vista, Australia). All signals were digitally recorded using a PowerLab system (16/35, ADInstruments, Bella Vista, Australia). Physiological and ultrasound data were synchronized using built-in triggers from the ultrasound scanner and PowerLab system.

\section{Cervical Magnetic Stimulation}

CMS of the phrenic nerves was performed using a $90 \mathrm{~mm}$ circular coil (1 Tesla maximum output) driven by a Magstim 200 stimulator (Magstim, Whitland, Dyfed, UK). The coil was placed on the spinous process of the seventh cervical vertebra. A series of stimulation at $100 \%$ of stimulator intensity was performed to detect the optimal stimulation spot (i.e. the spot where $\operatorname{Pdi}_{\mathrm{tw}}$ was maximal), the latter being skin-marked to ensure reproducible stimulation site throughout the protocol. Fig. 2 presents the experimental setup used for the experiment.

\section{E. Expiremental protocol}

CMS was performed from 30 to $100 \%$ of stimulator intensity, in a randomized order. Stimulations were delivered during an end-expiratory breath hold and lung volume at the onset of stimulation was checked to be consistent across trials. A minimum of three trials per intensity were completed, separated by $\sim 1$ min to avoid any effect of potentiation. Two to three validated trials were considered for further analysis.

\section{F. Data analysis and statistics}

Data were analyzed using standardized Matlab scripts (Mathworks, Natick, MA, USA). Pdi $i_{\mathrm{tw}}$ was computed as the difference between maximal Pdi value and Pdi at the onset of CMS. Diaphragm tissue velocity was calculated after a radiobased speckle tracking has been performed. A one-dimensional cross-correlations on two consecutive frames was used to measure the relative displacement of a pixel between the two frames [11]. Diaphragm tissue velocity was computed as the first-time derivative of pixel displacement and its maximal value $\left(\mathrm{Vdi}_{\max }\right)$ was confronted to $\mathrm{Pdi}_{\mathrm{tw}}$.

Because of non-normality, Friedman repeated measures ANOVA were used to evaluate the effect of stimulation intensity of $\mathrm{Pdi}_{\mathrm{tw}}$ and $\mathrm{Vdi}_{\max }$. Tukey's post-hoc were performed if a significant main effect of intensity was found. Spearman ( $\rho)$ correlation coefficients were calculated to analyze withinindividual relationships between the variables. Overall relationships were assessed using repeated measures correlations (R, [12]). Finally, absolute and relative within-day reliability of $\mathrm{Pdi}_{\mathrm{tw}}$ and $\mathrm{Vdi}_{\max }$ were assessed using standard error of measurement (SEM) and intraclass correlation coefficients (ICC), respectively.

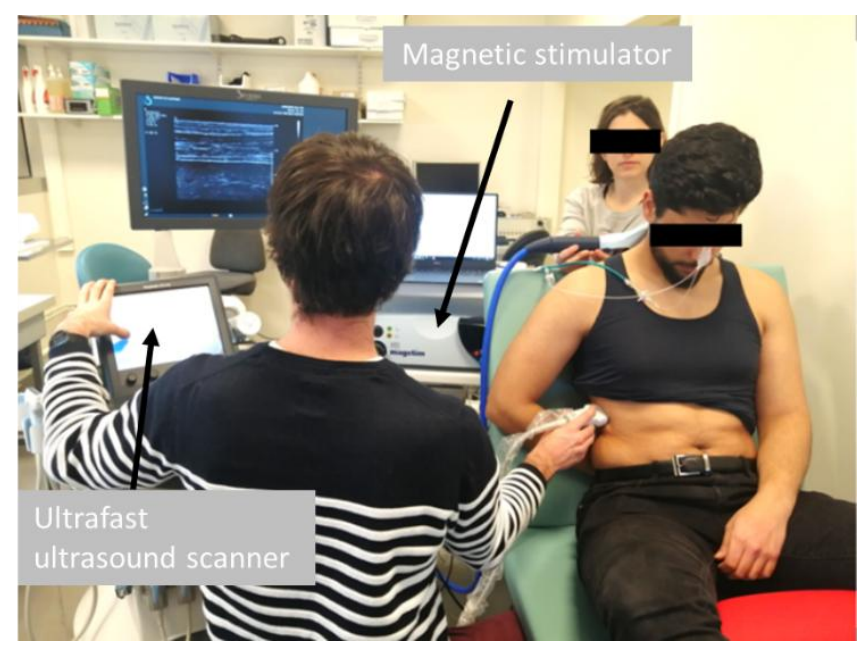

Fig. 2: Experimental setup. The subject is equipped with an esophageal and gastric balloon while being cervical magnetic stimulation is delivered on the optimal simulation spot. An ultrafast ultrasound scanner (Aixplorer, Supersonic Imagine, France) is used to image the zone of apposition of the right L...... 


\section{RESULTS}

\section{A. Ultrafast Ultrasound Imaging of the Diaphragm}

Fig. 1 displays a typical M-Mode image of the diaphragm during CMS. The diaphragm can be identified in-between the

\section{DisCUSSION \& PERSPECTIVES}

This work aimed at imaging the diaphragm during CMS at various intensity level. Our results show that diaphragm ultrasound-derived metrics are sensitive to stimulation intensity in healthy subjects. We also assessed if such ultrasound indices were related to diaphragm response during CMS (i.e. Pdi $\mathrm{tw}_{\mathrm{tw}}$ ).
A.

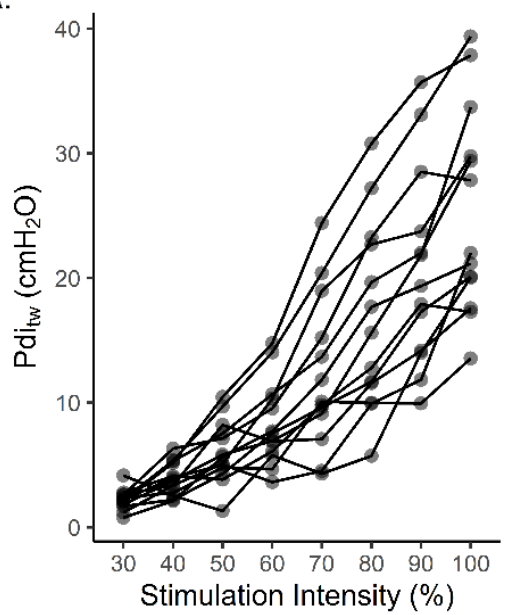

B.

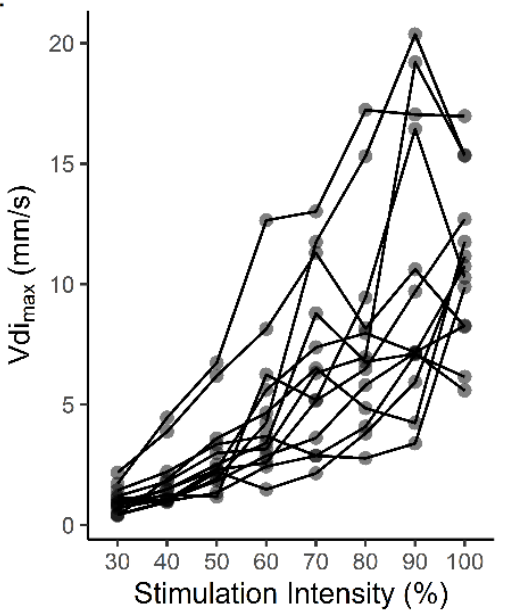

C.

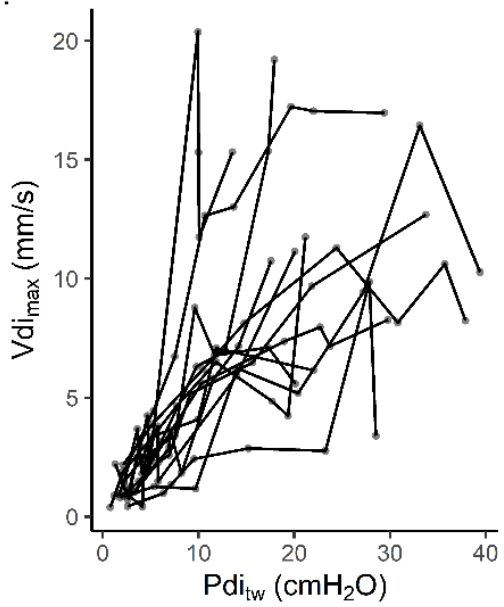

Fig. 3: Averaged data points for each participants regarding the relationships between stimulation intensity and twitch transdiaphragmatic pressure ( $\mathrm{Pdi}_{\mathrm{tw}}, \mathrm{A}$.), stimulation intensity and maximal diaphragm tissue velocity (Vdi $\mathrm{Vax}_{\text {max }}, \mathrm{B}$.) and between Pdi and $\mathrm{Vdi}_{\max }(\mathrm{C}$.

two hyper-echogenic layers surrounding the muscular layer.

\section{A. Relationships betwwen stimulation intensity, $P d i_{t w}$, and $V d i_{\text {max }}$}

All participants presented with a significant relationship between stimulation intensity and $\operatorname{Pdi}_{\mathrm{tw}}$ ( $\rho$ ranged from 0.83 to 1.00 , all $\mathrm{p}<0.01, \mathrm{R}=0.91, \mathrm{p}<0.01)$ and between stimulation intensity and $\mathrm{Vdi}_{\max }$ ( $\rho$ ranged from 0.79 to 1.00 , all $\mathrm{p}<0.05, \mathrm{R}$ $=0.83, \mathrm{p}<0.01)$. $\mathrm{Pdi}_{\mathrm{tw}}$ was significantly related to $\mathrm{Vdi}_{\max }$ in all participants ( $\rho$ ranged from 0.64 to 1.00 , all $p<0.05, R=0.75$, $\mathrm{p}<0.01$ ). Individual relationships between all variables are presented in Fig. 3.

Within-day reliability of $\mathrm{Pdi}_{\mathrm{tw}}$ and $\mathrm{Vdi}_{\max }$ are presented in Table 1.

Table 1: Standard error of measurement (SEM) and intraclass correlation coefficient (ICC) are presented jointly with the $95 \%$ confidence intervals for both twitch transdiaphragmatic pressure $\left(\mathrm{Pdi}_{\mathrm{tw}}\right)$ and maximal diaphragm tissue velocity $\left(\mathrm{Vdi}_{\max }\right)$.

\begin{tabular}{ccc}
\hline Variable & SEM & ICC \\
& {$[\mathbf{9 5}$ \% CI $]$} & {$[\mathbf{9 5} \% \mathbf{~ C I}]$} \\
\hline $\operatorname{Pdi}_{\mathrm{tw}}\left(\mathrm{cmH}_{2} \mathrm{O}\right)$ & $1.55[1.39 ; 1.75]$ & $0.97[0.96 ; 0.98]$ \\
& & \\
$\mathrm{Vdi}_{\max }\left(\mathrm{mm}^{-1} \mathrm{~s}^{-1}\right)$ & $1.89[1.70 ; 2.13]$ & $0.86[0.81 ; 0.90]$
\end{tabular}

We demonstrated that a strong relationship exists between $\mathrm{Vdi}_{\max }$ and $\mathrm{Pdi}_{\mathrm{tw}}$ in all participants.

In line with previous work $[7,8]$, we found that $\mathrm{Vdi}_{\max }$ increased with stimulation intensity in all participants. However, this work is the first to address the relationship between tissue velocity and the pressure/force it produces. Here, we demonstrated that $\mathrm{Vdi}_{\max }$ measured using ultrafast US was related to $\mathrm{Pdi}_{\mathrm{tw}}$ in all participants. This finding opens promising perspectives for clinical populations. Indeed, the routine monitoring of diaphragm function requires the use of aforementioned invasive esophageal and gastric balloons. Thanks to the relationship between Vdimax and $\mathrm{Pdi}_{\mathrm{tw}}, \mathrm{Vdi}_{\max }$ could potentially become a tool to monitor diaphragm function. More specifically, the relative increase or decrease in $\mathrm{Pdi}_{\mathrm{tw}}$ could be monitored via the relative increase or decrease in $\mathrm{Vdi}_{\max }$.

Future work should therefore focus on the use of ultrafast US in clinical populations. This technique may also be of interest in the detection of diaphragm dysfunction. Also, it may be noted that all measurements were performed by the same operator on a single day. Inter-operator as well as between-day reliability of $\mathrm{Vdi}_{\max }$ remains to be investigated.

\section{REFERENCES}

[1] Laveneziana, P., Albuquerque, A., Aliverti, A., Babb, T., Barreiro, E., Dres, M., ... \& Hudson, A. L. (2019). ERS statement on respiratory muscle testing at rest and during exercise. European Respiratory Journal, $53(6)$.

[2] Goligher, E. C., Laghi, F., Detsky, M. E., Farias, P., Murray, A., Brace, D., ... \& Ferguson, N. D. (2015). Measuring diaphragm thickness with 
ultrasound in mechanically ventilated patients: feasibility, reproducibility and validity. Intensive care medicine, 41(4), 642-649.

[3] Oppersma, E., Hatam, N., Doorduin, J., Van Der Hoeven, J. G., Marx, G., Goetzenich, A., ... \& Bruells, C. S. (2017). Functional assessment of the diaphragm by speckle tracking ultrasound during inspiratory loading. Journal of applied physiology, 123(5), 1063-1070.

[4] Bachasson, D., Dres, M., Nierat, M. C., Doorduin, J., Gennisson, J. L., Hogrel, J. Y., \& Similowski, T. (2018, October). Ultrafast Ultrasound Imaging Grants Alternate Methods for Assessing Diaphragm Function. In 2018 IEEE International Ultrasonics Symposium (IUS) (pp. 1-4). IEEE.

[5] Poulard, T., Fosse, Q., Hogrel, J. Y., Niérat, M. C., Similowski, T., Dres, M., Bachasson, D., \& Gennisson, J. L. (2019, October). Ultrasound shear wave elastography for assessing diaphragm function within the intensive care unit. In 2019 IEEE International Ultrasonics Symposium (IUS) (pp. 966-969). IEEE.

[6] Hager (j'attends que ce soit publié pour avoir la vraie citation sans le "in press"

[7] Deffieux, T., Gennisson, J. L., Tanter, M., \& Fink, M. (2008). Assessment of the mechanical properties of the musculoskeletal system using 2-D and 3-D very high frame rate ultrasound. IEEE transactions on ultrasonics, ferroelectrics, and frequency control, 55(10), 2177-2190.

[8] Grönlund, C., Claesson, K., \& Holtermann, A. (2013). Imaging twodimensional mechanical waves of skeletal muscle contraction. Ultrasound in medicine \& biology, 39(2), 360-369.

[9] Rossi, S., Hallett, M., Rossini, P. M., \& Pascual-Leone, A. (2011). Screening questionnaire before TMS: an update.

[10] Montaldo, G., Tanter, M., Bercoff, J., Benech, N., \& Fink, M. (2009). Coherent plane-wave compounding for very high frame rate ultrasonography and transient elastography. IEEE transactions on ultrasonics, ferroelectrics, and frequency control, 56(3), 489-506.

[11] Loupas, T., Powers, J. T., \& Gill, R. W. (1995). An axial velocity estimator for ultrasound blood flow imaging, based on a full evaluation of the Doppler equation by means of a two-dimensional autocorrelation approach. IEEE transactions on ultrasonics, ferroelectrics, and frequency control, 42(4), 672-688.

[12] Bakdash, J. Z., \& Marusich, L. R. (2017). Repeated measures correlation. Frontiers in psychology, 8, 456. 